\title{
How to Make Physical Activity Promotion Work in Thai Schools: Perspectives of Multiple Stakeholders
}

\author{
Areekul Amornsriwatanakul, Narongsak Noosorn, Kittipong Poonchob, Rung Wongwat, \\ Somkiat Sornprasit, Phataraphon Markmee, Michael Rosenberg, and Karen Milton
}

\begin{abstract}
Background: This study aimed to determine current practice in physical activity (PA) promotion in Thai schools, explore barriers and facilitators to PA promotion within the school setting, and identify strategies to support schools' future practice. Methods: A qualitative study design was applied comprising document analysis, focus groups, in-depth interviews, and onsite observations. The focus groups and in-depth interviews were conducted with 144 informants, purposively recruited through 24 schools in 4 regions across Thailand. Inductive content analysis was used. Results: Most schools were promoting PA in the absence of written policies, and without an accurate understanding of PA. Nonalignment of school performance indicators and policies, concerns about children's academic performance, and lack of budget were raised as barriers to PA promotion, whereas strong partnerships with multiple local stakeholders facilitated school-based PA promotion. Mandated government PA policies and more information support were suggested as strategies to enhance schools' future practices. Conclusions: For PA promotion to be successful in the school setting, significant challenges will need to be addressed. Results from this study help the government and concerned stakeholders to better understand the situation at the school level, and further strive for achieving the target PA levels specified in the National PA Plan.
\end{abstract}

Keywords: policy, implementation, health promotion, Thailand, youth, adolescent, public health practice

Regular participation in physical activity (PA) provides a wide range of physical, psychological, and social health benefits for children and youth. ${ }^{1}$ These benefits include short- and long-term improvements in cognitive function, concentration, and educational performance. ${ }^{2}$ However, recent evidence shows that globally a high proportion of children and youth $(67 \%-73 \%)$ do not participate in an average of at least 60 minutes of moderate to vigorous PA per day as recommended by the global PA guidelines. ${ }^{3}$ Levels of inactivity among children and youth are high in developed countries, as well as in developing countries such as Thailand.

The word "physical activity" is relatively new in Thailand, as well as in many other low- and middle-income countries. PA is primarily recognized through specific activity domains, particularly exercise and sports. However, many attempts have been made by several authorities to increase PA levels in Thai children. In 2018, Thai Ministry of Public Health launched a National PA Plan to increase the proportion of people who meet the global PA guidelines to $75 \%$ by $2030 .{ }^{4}$ The National Committee also adopted an "active children" policy to support this endeavor, which was consistent with the "active people" approach suggested by the Global Action Plan on PA 2018-2030. ${ }^{5}$ Thai Health Promotion Foundation has also implemented actions to promote active play in schools for many years, such as the "come out and play" and "active play" programs. ${ }^{6}$

Schools have been identified as a key setting for the delivery of population-wide PA promotion, as they provide almost universal

Amornsriwatanakul and Poonchob are with the College of Sports Science and Technology, Mahidol University, Salaya, Thailand. Noosorn, Wongwat, Sornprasit, and Markmee are with the Faculty of Public Health, Naresuan University, Phitsanulok, Thailand. Amornsriwatanakul and Rosenberg are with the School of Human Sciences (Sport Science, Exercise and Health), University of Western Australia, Perth, WA, Australia. Milton is with the Norwich Medical School, University of East Anglia, United Kingdom. Noosorn (nnoosorn@yahoo.com) is corresponding author. access to a majority of children and youth. ${ }^{7}$ Schools are influential in cultivating lifelong PA habits in children, as this age group spends a large amount of their waking time at school. In this regard school PA policies and their implementation are considered crucial to the success of promotional efforts to increase children and youth's PA levels. ${ }^{8}$

School PA policies can define priority areas, guide the delivery of school PA program implementation, and identify targeted outcomes. ${ }^{9}$ A previous study disclosed that a majority of Thai schools $(>80 \%)$ reported having many policies in place to promote PA among students, including physical education (PE), standard school sport facilities, and extracurricular activities. Nevertheless, these policies had no association with students' overall PA. ${ }^{10}$ These findings suggested that if Thailand is to be successful in raising children and youth's PA levels through the school setting, a greater understanding in what the policies comprise and how school PA policies are implemented is required.

The complexity of school governing systems can make it challenging to advocate for PA in schools. School policy formulation and implementation operate at different levels are multifaceted, and involve multiple stakeholders. Like many other countries, the Thai Ministry of Education (MOE) is the main agency supervising education at all levels and types (formal and informal). The formal education system presents an opportunity to reach $83 \%$ of school-age children (10.8 million). ${ }^{11}$ Under the MOE, the Office of the Basic Education Commission is responsible for the administration of the majority of the government's elementary and secondary schools across the country (78\%). The MOE coordinates this school administration through its 18 regional education offices covering all 77 provincial offices. ${ }^{12}$ As displayed in Figure 1, the remaining schools are managed by different government authorities within and outside the MOE.

At the school level, the principals employed by the MOE are assigned to oversee a school for a 4-year term, after which they are 


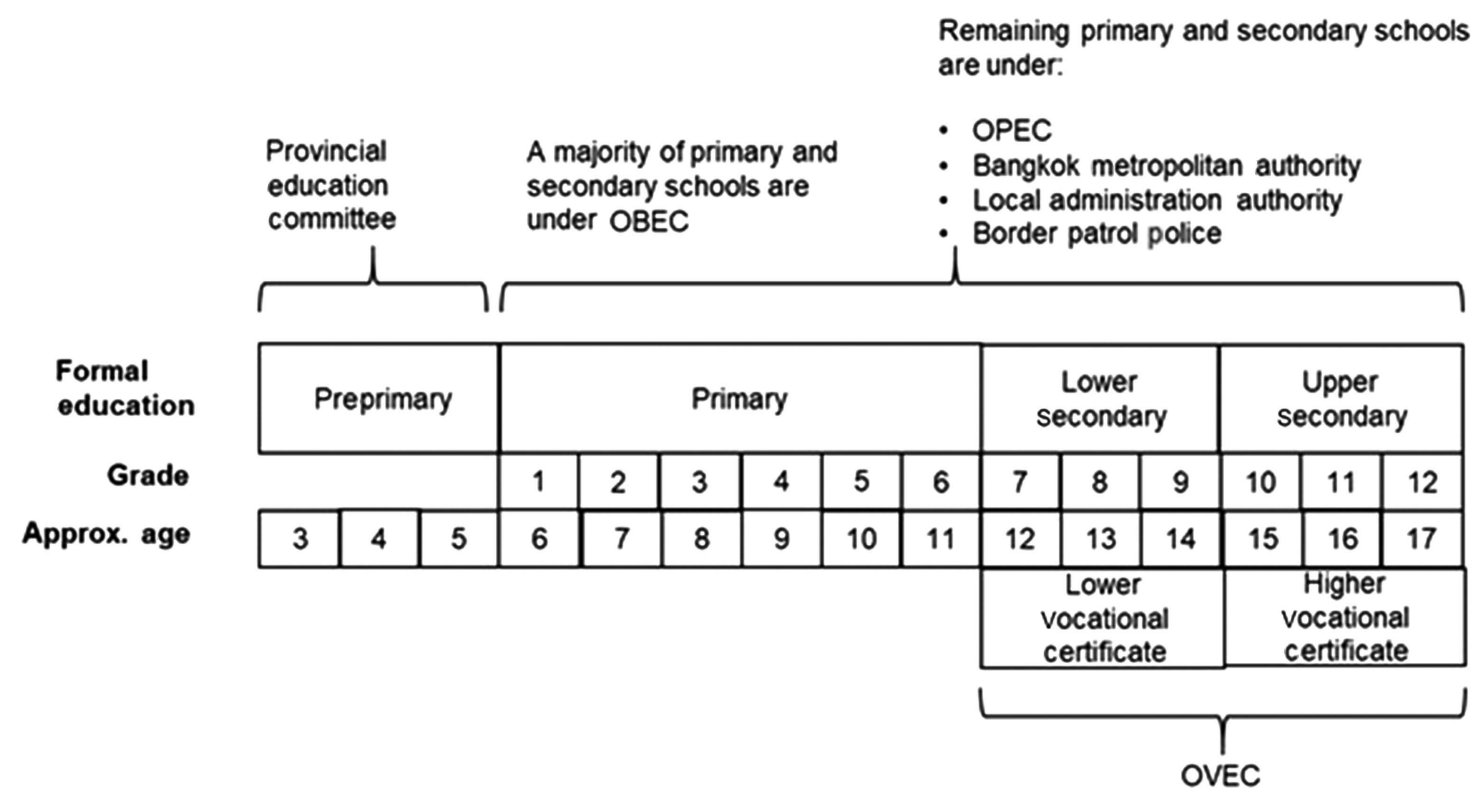

Figure 1 - Thai formal education system (adapted from Bureau of International Cooperation-Ministry of Education ${ }^{12}$ ). OBEC indicates Office of the Basic Education Commission; OVEC, Office of the Vocational Education Commission; OPEC, Office of the Private Education Commission.

assigned to another school. All schools in the country providing basic education follow the Basic Education Core Curriculum B.E. 2551 (2008), which broadly guides the contents and time allocation for the provision of all educational disciplines. ${ }^{13}$ At present, there are 5 policies that are directly or indirectly related to PA promotion in schools. PE and standard school sport facilities are the 2 compulsory policies required for all schools. Third is an active learning policy, recently introduced and promoted in all schools. Fourth is a policy on shared use of school sport facilities with the community during after school hours. Finally, the Health Promoting Schools project implemented under the national school health policy of the Ministry of Public Health, broadly addresses physical health in general. The last 3 policies are adopted and implemented on a voluntary basis.

Little prior research provides detail on how school PA policies are implemented, ${ }^{8}$ and the information is scarce in Thailand. Currently available implementation studies assess whether schools adopt international and/or national PA policies, the degree to which implementation complied with the policies/plans, and barriers and facilitators to implementation. ${ }^{14-18}$ Other literature/documents only suggest general frameworks on how to plan and implement policies to promote PA in the school setting. ${ }^{19-21}$ Therefore, this study aimed to: (1) determine current practice in PA promotion in Thai schools, (2) explore barriers and facilitators to PA promotion within the school setting, and (3) identify strategies to support future practice.

\section{Methods}

To gain an in-depth understanding of current practice to promote PA in Thai schools, and the barriers and facilitators to PA promotion within the school setting, a qualitative study design was applied.

\section{Samples and Recruitment}

Geographically, Thailand was stratified into 4 regions (Central, North, South, and Northeastern), and each region was stratified further into small, medium, and large provinces based on their population size. ${ }^{22}$ A province was randomly selected from each stratum in each region, resulting in 12 provinces. From each province, the city district was purposively chosen, and a noncity district was randomly chosen. A school was then randomly chosen from the city district and the noncity district. From these stratifications, a total of 24 schools were invited to participate in this study.

Field research staff contacted the schools and provided them with a document package, including an invitation letter, information sheet, a list of interview questions, an example of the consent form, and a copy of the certificate from the ethics committee. All invited schools accepted the invitation and were requested to help recruit 6 informants: (1) school principal, (2) student affair teacher, (3) class teacher, (4) PE teacher, (5) parent/guardian or member of school executive board, and (6) student. They were purposively invited to participate in this study based on their involvement in policy implementation at the school level. In total, 144 informants participated in the study, and all participants took part in both a focus group and an in-depth interview.

\section{Data Collection Methods and Protocols}

Data collection involved the following: (1) reviews of schools' policy documents and strategic plans, (2) face-to-face focus group discussions, (3) face-to-face in-depth interviews, and (4) onsite observations including taking photographs of school PA-related facilities.

Based on Daly-Smith et $\mathrm{al}^{23}$ and the extensive experience of 2 coauthors (A.A. and K.P.) in the PA and management fields, a conceptual framework of how schools implement their PA policies and factors potentially influencing implementation of the policies was depicted (Figure 2). The framework helped to ensure that all research team members shared the same concept, and this assisted the development of 2 semi-structured question guides, one for the focus groups and one for the interviews.

Schools could provide their strategic or operational plan to the research team on the data collection day or send the electronic file(s) beforehand. Due to the geographical spread of schools and resource constraints, all other data collection methods were 


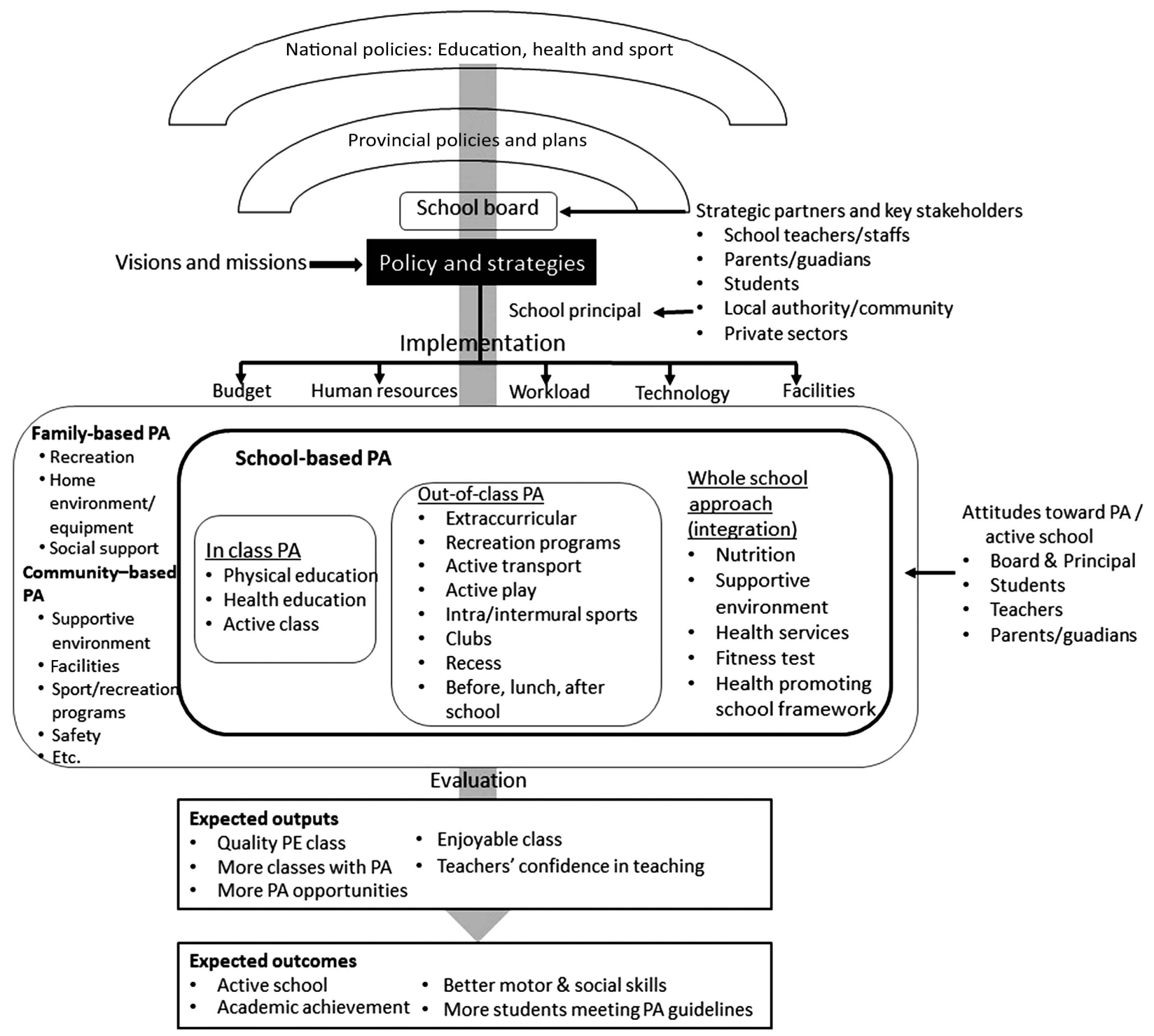

Figure 2 - Conceptual framework of school PA policy implementation (adapted from Daly-Smith et al ${ }^{23}$ ). PA indicates Physical activity.

conducted on the same day at each participating school. On the data collection day, the focus group discussion was conducted first, followed by the in-depth interview with each individual informant, and finally, the onsite observation. The same data collection methods and protocols were applied in all schools. The whole data collection process at each school took approximately 5 to 6 hours. Data were collected from August to October 2020 (postCOVID-19 lockdown policy).

\section{Reviews of Schools' Policy Documents and Strategic Plans.}

All schools readily had their annual strategic plans available as they had to regularly report to the MOE. The schools' 2020 strategic plans were reviewed before or after the data collection day (depending on the school's submission). The key information needed was the written PA policy statement(s) and strategies or action plans relevant to the policies.

Face-to-Face Focus Group Discussions. The focus group discussions were used to clarify the roles of each informant and explore the relationships among the informants and group dynamics. The 6 informants (one from each category) recruited through the school participated in the discussion, which was arranged at a school's meeting room. During the discussion, the principal investigator acted as a moderator, facilitating the focus group discussion. Other research team members $(n=5)$ were assigned a specific role, that is, moderator assistant, question checker, notetaker, and observer. High-level open-ended questions were asked, for example, hat policies did the school have in relation to PA promotion? How did the school implement the policies? Who were involved in the process? What were their roles? The focus group discussion took approximately 2 to 3 hours.

Face-to-Face In-Depth Interview. The in-depth interviews were used to gain insights from each informant on PA promotion in schools and their involvement in and/or contributions or support to policy implementation. The in-depth interviews were also arranged at the school's meeting rooms or in a nearby area depending on the school's space. The principal investigator interviewed the school 
principal. Thai coauthors and trained research staff interviewed other informant categories. The interviews sought information on the informants' jobs and roles in the policy implementation process, activities arranged, and barriers and facilitators to PA policy implementation. The interview took approximately 1.5 to 2 hours.

Onsite Observation Including Taking Photographs of School PA-Related Facilities. Data from the onsite observation were used to verify the data collected from the focus groups and interviews. Field research staff were assigned to take photographs of school PArelated facilities and sport/exercise equipment storage. The observation was accompanied by teachers or other school personnel. The photo taking focused on the facilities and avoided capturing students, due to ethical considerations. The digital photographs were saved and classified according to school name. They were viewed subsequently by researchers during data analysis. The onsite observation took approximately 1 hour at each school depending on the school size.

This study protocol received ethical approval from Mahidol Central Institutional Review Board (MU-CIRB 2020/104.0405). Written consent was obtained from all participants included in this study.

\section{Data Analysis}

Digital voice recordings were transcribed verbatim without mentioning informants' names, and randomly checked by the moderators/interviewers to ensure accuracy of the data. We decided not to use any available data analysis software as they did not work well in the Thai language. Twelve researchers in the team worked in pairs to analyze the transcripts. Each pair was responsible for the same informant category they conducted in-depth interviews for. Data were analyzed between October 2020 and January 2021, using an inductive method of open coding to allow broad concepts and patterns to emerge. Thematic codes were grouped into larger categories or subthemes, and subsequently, main themes. All researchers were required to maintain their audit trails to be able to trace back their decision making and ensure a rigorous analysis.

Interpretations of the transcripts and classification of codes into each main theme were supported by informants' quotes or other relevant evidence; for example, policy statements, strategic plan, and photos. Each pair of researchers discussed any disagreements on the themes developed between them before presenting them in research team meetings. The presented themes were challenged, and they were discussed and verified among all researchers to minimize bias. To help verify the main themes, 3 senior researchers (A.A., K.P., and N.N.) reviewed all the transcripts $(n=168 ; 24$ focus groups and 144 interviews) and school policy documents or strategic plans (24 schools) and discussed any disagreements until they reached a consensus. This data analysis approach was adapted from Marshall and Rossman. ${ }^{24}$

Draft results and recommendations of this study were presented in an online meeting in April 2021 among the school principals participating in this study. They reviewed the results and commented on the recommendations. Their constructive comments were considered when producing this manuscript.

\section{Results}

\section{Characteristics of Schools Participating in the Study}

Participating schools were equally distributed across geographical regions. Most schools (87.5\%) were under the supervision of the
Table 1 Characteristics of the 24 Participating Schools

\begin{tabular}{lc}
\hline School characteristics & N (\%) \\
\hline School geographical location & $6(25.0)$ \\
$\quad$ Central & $6(25.0)$ \\
North & $6(25.0)$ \\
South & $6(25.0)$ \\
$\quad$ Northeastern & \\
School district & $12(50.0)$ \\
City district & $12(50.0)$ \\
Noncity district & \\
School governing system & $21(87.5)$ \\
Office of the basic education commission & $3(12.5)$ \\
Local/provincial authority & \\
Education level offered & $7(29.2)$ \\
Kindergarten to junior secondary (K1-grade 9) & $1(4.1)$ \\
Primary to junior secondary (grades 1-9) & $16(66.7)$ \\
Secondary (grades 7-12) & \\
Size of schools & $14(58.33)$ \\
Small-medium (<1500 students) & $10(41.67)$ \\
Large ( $\geq 1500$ students) & $1(4.17)$ \\
School written physical activity policies/strategies & $23(95.83)$ \\
Yes & \\
No & \\
\hline
\end{tabular}

MOE's Office of the Basic Education Commission, and more than half $(66.7 \%)$ were secondary schools. Almost $60 \%$ of schools had $\leq 1500$ students, and almost all schools (96\%) had no official written PA policies or strategies as shown in Table 1.

All schools that participated in this study implemented the MOE's policies on the provision of PE class and standard school sport facilities. All schools complied with the minimum requirements of both policies guided by the ministry (ie, provision of 25 min of PE class/week). Most schools were trying to adjust their teaching techniques to follow the MOE's policy on active learning. All schools had experience in joining the Health Promoting Schools project of the Ministry of Public Health. None of the participating schools initiated their own policies to specifically promote PA, except one school located in a noncity district in the Northern region. The principal of this school shared that the school's strategic plan clearly states that there must be a project to promote PA or exercise among students.

The qualitative findings are grouped into 4 main themes: current practice in PA promotion in schools, barriers to PA promotion within the school setting, facilitators to PA promotion within the school setting, and strategies to support schools' future practice.

\section{Current Practice in PA Promotion in Schools}

Promoting PA Without Knowing It. The word "Physical Activity" in Thai is relatively new and was complicated for participants to understand. Researchers needed to explain the term and used the words "sport" or "exercise" interchangeably with PA to facilitate the conversation. School principals and teachers realized subsequently that they were promoting a number of physical activities at school without knowing that those activities promoted physical 
movements. The activities included, for example: games/play (that required movement), bike to study at partnering university, cleaning day, occupational training (eg, planting or agricultural activities and construction), boy or girl scouts; participation of students in community ritual ceremonies, traditional dancing and musical performances, and musical instrument marching rehearsals or competitions. Many principals and teachers expressed enthusiasm and pride, for instance, one said ". . . actually, we are doing a lot here in our school. We strongly support students' 'Pong Lang' plays, which is very famous in this area and neighboring provinces. People know it well." "Pong Lang" is a traditional Thai musical performance in which players dance fast while playing the musical instrument. Performers who play other musical instruments in the band also dance along.

Promoting PA in Absence of Written School PA Policies. None of the informants were aware of the National PA Plan. They had never heard of the World Health Organization's PA guidelines and were unaware of the national PA recommendations. Most schools (principals and teachers) reported that they did not have any written policies designed specifically to promote PA in their school, in addition to the policies imposed by the government. However, schools provided support to their students through sport activities, competition, and clubs, as well as other PA-related activities. One principal said "Well, our strategic plan only addresses health in general. We do not spell it [PA promotion] out clearly in the plan, but we provide a lot of support to students' activities." and another principal mentioned "We don't have that [PA] policy . . . every activity must be outlined in the [school strategic] plan in order to get the budget for the implementations." Therefore, an "absence of the policies, but a lot of actions" is a typical scenario for most Thai schools concerning PA promotion.

\section{Barriers to PA Promotion Within the School Setting}

Nonalignment of School Performance Indicators and Policies. Informants described inconsistencies between a set of school performance indicators of the MOE and its expectations for schools to also comply to the policies of other ministries. Informants raised that while schools had to comply with the MOE's education policies, they also had to collaborate with other government authorities, as well as provincial and local administrations, to handle many other issues such as elections, and community social, and cultural issues. However, the set of indicators used to evaluate schools' performance did not include any criteria recognizing schools' extra efforts or active engagement in other policies and issues. One principal explained "They [the government] wanted us to implement this and that ... eventually they used the results from $\mathrm{O}-\mathrm{NET}$ to assess if we are doing a good job. There are no reasons why we need to do more [than we are officially required]." O-NET is the Ordinary National Educational Test, which students in grades 6,9 , and 12 have to take to examine their academic proficiency.

\section{Serious Concerns About Students' Academic Performance.}

Parents' concerns about their child's academic performance were raised as the most critical factor for any school's decisions. Teachers and parents, including school executive board members, highlighted that it was crucial to maintain parents' confidence that their child's academic performance would not be negatively affected by any of the school's policies or activities. Principals and teachers expressed concerns about schools being required to promote PA and/or adopt an active school policy. One of them commented "We all know that exercise is important to children's health and good for their brain, but parents are more concerned about children's grades." Informants found that it was not easy to convince parents about the benefits of PA, as one teacher shared "Some parents called me and complained why school got their child to do things other than studying. Some of them even offered to pay or make a donation to school in exchange for their child's involvement in other activities."

Inadequate Budgetary Support. Many principals and teachers, particularly from small schools or schools located in the noncity districts, indicated that their school did not have an adequate budget to support PE and PA (Government budget is allocated to schools based on the number of students). Frequently raised issues were inadequate spaces or sport fields, and sport/exercise equipment. Some schools had sport/exercise facilities, but they were not in good condition. One informant mentioned "We need budget and equipment for our activities. We have soccer and petanque fields, but they do not meet the standard." One PE teacher expressed similarly "There are 30-40 students in each class. The number of sport equipment is limited; for example, we have only 4-5 futsal balls. Some students in the class might not have a chance to touch the ball." Another added that "We had a project to teach students to swim but we had no swimming pool and needed to ask for help from other organizations .... Sport equipment are also not enough for our students . . . we want more (budget) . . . ." One student expressed "[I] want the school to have more equipment and space for various activities such as sport field. It is currently not convenient for exercise . . . " This information is supported by photos taken at the schools, which generally showed poor facilities and a lack of good quality equipment.

\section{Facilitators to PA Promotion Within the School Setting}

Strong Partnership With Multiple Local Stakeholders. Schools had a good connection with public and private organizations including state enterprises and not-for-profit organizations. In addition, schools had a close connection with guardian association, school alumni, and their community. Schools received strong support from these stakeholders in many forms, including in-cash and in-kind. Examples of support were given by many informants. First, "Our school received an opportunity from the city, sub-district administration, community leader, health village volunteers, head of the village. They provided financial support to our school to buy equipment for our organized activities." Second, "Local Administration, temples, communities, police stations, and Sport University in our province are kind to support athletic fields as we do not have that. We took our students to use their facilities." Third, "We work with Sport Associations . . . and ... Provincial Public Health Office and Thai Health Promotion Foundation. In light of the activities concerning environmental issues, we work with Environment Institute, Department of Environmental Quality Promotion, YMCA and Rotary clubs." Finally, "Parents support equipment or sport clothes or water when we organized the activities."

\section{Strategies to Support Schools' Future Practice}

Mandated and Sustainable Policies Preferred. Most school principals and teachers, and a majority of representatives of school 
executive boards, preferred mandated policies. One principal raised "We want them [the MOE] to specify the policies clearly and apply the same across the country." Continuity and sustainability of the policies was expressed as important by most schools, as reflected by one principal: "The Ministry [of Education] does it intermittently . . . we managed whatever they wanted, but it was not promoted or supported on a regular basis. It usually comes for a while and go ....."

Mandated policies were preferred because informants felt that they needed institutional support, and particularly a clear rationale underpinning each policy, approach, or guideline in order to support and encourage implementation. In addition, the equitable application of policies in all schools across the country would help schools in dealing with parents' or guardians' questions and complaints. One teacher expressed that "If the ministry does it all the same, parents would not complain." Another suggested "We not only want a top-down or mandated policy, but also guidance and supporting tools or examples of how we can promote PA in our class. We also want to know how PA can get along with subjects' technical contents."

More Informational Support Needed. Informants suggested that public campaigns would raise awareness among teachers and parents, as well as the general public about the importance of PA and how to integrate PA into their daily lives. One principal pointed out "Responsible authorities should publicize the fact that PA is beneficial to children's learning ability." Most informants understood that PE was the only class related to PA. When they participated in the focus group discussion, they learned what PA is and realized that PA could be applied to other classes. However, they needed guidance to integrate PA into their classes, especially at the beginning. As an example, one teacher mentioned "I can't really imagine how I can use PA in my Thai class." Robust scientific evidence, particularly local experiments which are conducted in Thai children and contexts, would help parents/guardians to be less worried about the negative effects of PA (or exercise and active play) on their children's academic performance. One of the principals raised "We need strong evidence to show to the guardians that physical activities or exercise help improve their child's academic outcomes."

\section{Discussion}

This study set out to explore current practices to promote PA in Thai schools, barriers and facilitators to school PA promotion, and strategies to overcome challenges to PA promotion in the school setting. Based on the qualitative content analysis of key stakeholders' perspectives and experiences, PA was not well understood by stakeholders, received a low priority, and was lacking in policy support at the national, provincial, and school levels. The results of this study suggest there remains significant challenges that need to be considered when promoting school-based PA in Thailand.

The PA, like other health behaviors, operates across multiple levels within a complex system (Figure 2). At the national level, there exists the PA plan, although schools were unaware of its existence, of any policy direction, or guidance directed to the schools. At the provincial level, there are policies or plans to promote people's health in general, but mostly through the promotion of sports and exercise, rather than PA more broadly. At the school level, there were almost no specific PA policies or plans beyond the ones required by the MOE. All but one school had no strategic plans outlining actions and budgets dedicated directly to raising participation in exercise or PA among students.

School principals and teachers considered PA promotion as an unnecessary extra effort, as it was not formally evaluated as part of their performance. In part, this situation might have been expected in Thailand, as there was little understanding of the term "physical activity" at all levels. This contrasts practices in western countries, where PA occupies policy and practices in the governance of schools and is strongly supported by education departments. ${ }^{17,25}$ Nonetheless, Thai schools were offering their students opportunities to engage in a range of sport and recreational activities that could be classified as PA. Most of the organized activities were conducted outside of the classroom, and the types of activities were related to schools' local sociocultural contexts. The out-of-classroom opportunities for PA occurred sporadically depending on the preferences of the principal or responsible teachers who organized them, and consequently were unsustainable. PE was the only inclass opportunity for Thai students to be physically active, and for PA to be promoted. This finding is again contrasted by western countries where PA is mandated by the government and schools are required to deliver PA to all children. ${ }^{17,25}$ In the United States and Australia as examples, there is a legislation(s) at the national level aiming to promote students' wellness with a clear goal for PA. The Child Nutrition and Women, Infants, and Children Reauthorization Act of 2004 of the United States requires all education agencies participating in federal food programs to create a school wellness policy promoting healthy diet and PA. ${ }^{25}$ In Australia, the Schools Assistance Act 2004 (Section 14) obliges state funding for government schools to provide education with at least 2 hours of PA/ week. ${ }^{17}$ These examples from western countries reflect the significance of government policy support for PA promotion in the school setting. The mandated policies assist schools by guiding their efforts and plans of actions. ${ }^{26}$ Evaluation studies show efficacy of the mandated policies in improving school practices, as they ensure an alignment of the policies and a firm basis for implementation, including resources at all levels. ${ }^{17,25,27,28}$ Evidence also shows that mandated policies help address the equity and sustainability issue. ${ }^{28}$ In Thailand, it seemed clear that when PA policies were not mandatory and there were clashes between educational and health demands, schools/teachers inevitably chose to respond to educational outcomes rather than health-oriented outcomes. Thus, one way to help Thai schools to have written policies and address PA in school action plans in a sustainable manner is to have a mandated PA policy initiated at the national level, and this idea was supported by schools.

In the conceptual framework (Figure 2), attitudes of key stakeholders can also influence how PA operates and is promoted. In our case, parents' concerns about their child's academic outcome emerged and were anecdotally believed to influence policy decisions at all levels. Available literature shows that only $9 \%$ of Thai children engaged in active play for $>2$ hours per day, and $11.7 \%$ engaged in outdoor play of $\geq 2$ hours per day. ${ }^{29}$ The low levels of children's PA in Thailand may reflect the low priority of parents, teachers, and administrators for children to engage in regular PA. These findings potentially reflect cultural differences in Thailand compared with more developed western countries, where PA in children is prioritized by most of society. Evidence suggests that when Thai children receive parental support there is a greater chance for them to engage more in PA. ${ }^{30}$ However, parents in different cultures have different views toward education and PA. Parents in Thailand and many other countries in Asia (eg, China, Korea, Taiwan) enormously invest in their child's education; they 
reduce their spending on health and recreation or are financially in debt in order to give their child the best possible education. ${ }^{31}$ This is because, other than a reputable degree, there is no alternative pathway to success or a good profession, or in other words, high income. This belief is deeply embedded in Asian social values and culture. ${ }^{32}$ Consequently, parents' high hopes and the pressure placed on ensuring a good return on their investment is understandable. Schools hold the main responsibility for the educational outcomes of their students; thus their decisions and actions are susceptible to social values. This may explain why Thai schools prefer a mandated PA policy rather than the policy initiated at the school level. In western countries, PA has long been introduced to society before it was in Asia; PA receives a greater significance in people's lives, and has become part of their identity (ie, personal taste and lifestyle) and culture. Leisure-time PA receive equal or greater importance than studying, and this is particularly true in Europe. ${ }^{33}$ Therefore, educational system and schools must take responsibility for giving all children opportunities for daily PA and developing movement habits that can be carried through to adulthood. ${ }^{34}$ As discussed, Asian parents' attitude toward PA may need to be altered for school-based PA promotion to be successful. However, achieving a paradigm shift in social values and culture is highly challenging and takes time. Certainly, there is considerable work to be done for anyone attempting to increase PA among children in the Thai school system, on persuading parents of the value of PA for their children. Furthermore, how willing principals/ teachers, civil servants, and politicians will be to promote PA without strong parental support is questionable.

For Thailand and other countries with similar school governing systems and cultural contexts, we recommend lobbying for a mandated national PA policy to be implemented in all schools. Otherwise, as the results of this study suggest, it is difficult to be consistent and successful in raising children's PA levels. Communication efforts should be made from national down to school level to disseminate the existing national PA plan, and to increase public understanding about the concept of PA. Training programs for principals and teachers should be considered to enhance PA literacy. Further investigation is required into how to shift the cultural value of PA and children's health among the Thai population to value regular PA as an important contributor to achieving developmental milestones. Additional research is warranted on developing effective guidance or tools to support school policy development and implementation. Finally, the findings suggest that parental support for the value of PA in schools is critical to the success of any interventions. Therefore, there is important work to be done in convincing people, and particularly parents, in Thailand, and potentially other Asian countries, of the value of PA. Therefore, further investigation into how to gain parental support, including studies on the positive impact of PA/exercise on children's academic performance should be conducted.

\section{Strengths and Limitations}

The present study has several strengths and limitations that need to be taken into consideration when utilizing the study's findings and recommendations. The sample size is relatively large, and the sample was equally distributed across all geographical areas. This large sample size and wide coverage helped ensure that the data were truly saturated and represented typical scenarios across the country. In addition, the results were triangulated and verified by various sources and approaches (ie, written evidence, photos, and key informants). However, the school samples recruited into the study were public schools only. Parents/guardians or members of the school executive board and students were recruited with assistance from school principals or administration. Therefore, their views may introduce bias in the data. Social desirability of the informants, their bias toward interviewers' appearance and personalities, and experiences and interpretation of the researchers are other possible limitations of qualitative studies.

\section{Conclusions}

The present study provided novel insights into schools' current practices in PA promotion, factors hindering or facilitating PA policy implementation, and strategies to make PA policy work in Thai schools. Results suggested several challenges that were interrelated to each other in the Thai school educational system and culture. Results from this study can help the government and concerned stakeholders to better understand the situation at the school level and why PA efforts yield limited success. For the country to successfully reach the national PA target levels by 2030 , the findings from the present study should be considered when initiating or revitalizing efforts to promote PA in the school setting.

\section{Acknowledgments}

The authors feel gratitude to all school principals, teachers, parents/guardians, and students who participated in this study. The authors would also like to thank Children and Youth PA Studies and Naresuan University staffs who assisted in data collection and analysis. This study was financially supported by Thai Health Promotion Foundation through the Office of Children and Youth Physical Activity Studies. The sponsor was not involved with nor had any roles regarding the conduct of this study and publication.

\section{References}

1. Poitras VJ, Gray CE, Borghese MM, et al. Systematic review of the relationships between objectively measured physical activity and health indicators in school-aged children and youth. Appl Physiol Nutr Metab. 2016;41(6):S197-S239. doi:10.1139/apnm-2015-0663

2. Esteban-Cornejo I, Tejero-Gonzalez CM, Sallis JF, Veiga OL. Physical activity and cognition in adolescents: a systematic review. $J \mathrm{Sci}$ Med Sport. 2015;18(5):534-539. PubMed ID: 25108657 doi:10. 1016/j.jsams.2014.07.007

3. Guthold R, Stevens G, Riley L, Bull F. Global trends in insufficient physical activity among adolescents: a pooled analysis of 298 population-based surveys with 1.6 million participants. Lancet Child Adolesc Health. 2020;4(1):23-35. PubMed ID: 31761562 doi:10. 1016/S2352-4642(19)30323-2

4. Department of Health. National Physical Activity Promotion Plan (2018-2030). Ministry of Public Health; 2018.

5. Department of Health. Physical Activity Policies Proposed by the National Sub-Committee of the National Action Plan on Physical Activity. Nonthaburi, Thailand: Ministry of Health; 2019:66.

6. Thai Health Promotion Foundation. ThaiHealth Master Plan 2018 2020. Bangkok, Thailand: Thai Health Promotion Foundation; 2018.

7. World Health Organization. Global Action Plan on Physical Activity 2018-2030: More Active People for a Healthier World. Geneva, Switzerland: World Health Organization; 2018.

8. Lounsbery MAF, McKenzie TL, Smith NJ. School physical activity policy. Transl J Am Coll Sports Med. 2019;4(17):173.

9. Bull FC, Bellew B, Schöppe S, Bauman AE. Developments in National Physical Activity Policy: an international review and 
recommendations towards better practice. J Sci Med Sport. 2004;7(1):93-104. doi:10.1016/S1440-2440(04)80283-4

10. Amornsriwatanakul A, Lester L, Rosenberg M, Bull F. School policies and practices associated with Thai children's overall and domain specific physical activity. PLoS One. 2021;16(1):e0245906. PubMed ID: 33481937 doi:10.1371/journal.pone.0245906

11. Office of the Permanent Secretary-Ministry of Education. 2016 Educational Statistics. Bangkok, Thailand: Bureau of Information and Communication Technology; 2016.

12. Bureau of International Cooperation-Ministry of Education. Thai Education in Brief. 2017. https://www.bic.moe.go.th/images/stories/ pdf/thai_education_in_brief_2017.pdf. Accessed October 5, 2020.

13. Ministry of Education. National Core Curriculum for Basic Education. Bangkok. 2008.

14. Nathan N, Elton B, Babic M, et al. Barriers and facilitators to the implementation of physical activity policies in schools: a systematic review. Prev Med. 2018;107:45-53. PubMed ID: 29155228 doi:10. 1016/j.ypmed.2017.11.012.

15. Mâsse LC, Naiman D, Naylor P-J. From policy to practice: implementation of physical activity and food policies in schools. Int $J$ Behav Nutr Phys Act. 2013;10(1):71. doi:10.1186/1479-5868-10-71

16. Gallego P, Galán I, José Medrano M, Ramos P, Rivera F, Moreno C. Recent changes in school-based policies on physical activity and nutrition in Spain. Eur J Public Health. 2014;24(6):899-904. PubMed ID: 24614650 doi:10.1093/eurpub/cku022

17. Stylianou M, Walker JL. An assessment of Australian school physical activity and nutrition policies. Aust N Z J Public Health. 2018;(1):16. doi:10.1111/1753-6405.12751

18. Haug E, Torsheim T, Samdal O. Local school policies increase physical activity in Norwegian secondary schools. Health Promot Int. 2010;25(1):63-72. PubMed ID: 19884244 doi:10.1093/heapro/ dap040

19. World Health Organization. School Policy Framework: Implementation of the WHO Global Strategy on Diet, Physical Activity and Health. Geneva, Switzerland: World Health Organization; 2008.

20. World Health Organization. Promoting Active Living in and Through Schools: Policy Statement and Guidelines for Action. Esbjerg, Denmark: World Health Organization; 2000.

21. Center for Disease Control and Prevention. Increasing Physical Education and Physical Activity: A Framework for Schools. Atlanta, GA: US Department of Health and Human Services; 2017.
22. Department of Provincial Administration. Population and household statistics. 2020. https://stat.bora.dopa.go.th/stat/statnew/statyear/\#/ TableTemplate/Area/statpop. Accessed June 30, 2020.

23. Daly-Smith A, Quarmby T, Archbold VSJ, et al. Using a multistakeholder experience-based design process to co-develop the Creating Active Schools Framework. Int J Behav Nutr Phys Act. 2020;17(1):13. PubMed ID: 32028968 doi:10.1186/s12966-020-0917-z

24. Marshall C, Rossman G. Designing Qualitative Research. 3rd ed. London, UK: Sage; 1999.

25. Schwartz MB, Henderson KE, Falbe J, et al. Strength and comprehensiveness of district school wellness policies predict policy implementation at the school level. J Sch Health. 2012;82(6):262-267. PubMed ID: 22568461 doi:10.1111/j.1746-1561.2012.00696.x

26. Lounsbery M, McKenzie T, Morrow J, Monnat S, Holt K. District and school physical education policies: implications for physical education and recess time. Ann Behav Med. 2013;45:131-141. doi:10. 1007/s12160-012-9427-9

27. Steven HK, Andrew SS, Cristina SB, et al. Implementation of Texas senate bill 19 to increase physical activity in elementary schools. $J$. Public Health Policy. 2009;30:S221. doi:10.1057/jphp.2008.64.

28. Robertson-Wilson JE, Dargavel MD, Bryden PJ, Giles-Corti B. Physical activity policies and legislation in schools: a systematic review. Am J Prev Med. 2012;43(6):643-649. PubMed ID: 23159260 doi:10.1016/j.amepre.2012.08.022

29. Amornsriwatanakul A, Nakornkhet K, Katewongsa P, et al. Results from Thailand's 2016 report card on physical activity for children and youth. J Phys Act Health. 2016;13:S291-S298. PubMed ID: 27848741 doi:10.1123/jpah.2016-0316.

30. Amornsriwatanakul A, Lester L, Bull FC, Rosenberg M. Ecological correlates of sport and exercise participation among Thai adolescents: a hierarchical examination of a cross-sectional population survey. $J$ Sport Health Sci. 2020:1-14.

31. Kee-Cheok C, Christopher H, Yin-Ching L, Chen Z, Zheng Z. "Parents just don't understand"- -generational perceptions of education and work. High Educ Eval Dev. 2018;12(2):85-98.

32. Sharma Y. Asia's parents suffering 'education fever'. 2013. https:// www.bbc.com/news/business-24537487. Accessed May 20, 2021.

33. Engström L-M. Social change and physical activity. Food Nutr Res. 2004;48(3):108-113.

34. Alvarez-Bogantes CE. Understanding parents' perceptions of children's physical activity. Educare. 2019;23(2):306-323. 